\title{
Relación entre inteligencia emocional y optimismo vs. pesimismo en trabajadores de centros para personas con discapacidad intelectual
}

\author{
The relationship between emotional intelligence and optimism vs. \\ pessimism in workers of centers for people with intellectual disability
}

\begin{abstract}
Resumen
El uso adaptativo de las emociones en trabajadores que ejercen su profesión con personas con discapacidad intelectual resulta determinante para el ejercicio de su actividad cotidiana. Este estudio analiza la relación entre inteligencia emocional y optimismo vs. pesimismo. La muestra está compuesta por 59 sujetos $(n=59)$, con una edad media de 38,59 años $( \pm 10,359)$, pertenecientes a diferentes centros asistenciales. Se emplean los siguientes instrumentos: Trait Meta-Mood Scale-24 (TMMS-24), Emotional Quotient inventory (EQi-C) y Life Orientation Test Revised (LOT-R). Cabe destacar el empleo del coeficiente Omega por ser una adecuada medida de confiabilidad si no se cumple el principio de equivalencia. Las evidencias muestran la relación entre algunas de las dimensiones de los instrumentos considerados $(p<.05)$ y hay diferencias significativas a nivel estadístico entre las dimensiones de inteligencia emocional y la variable sociodemográfica género $(p<.05)$. El análisis de regresión confirma la asociación entre inteligencia emocional (reparación) con optimismo e inteligencia emocional (manejo del estrés) con pesimismo.
\end{abstract}

\section{Palabras clave}

Discapacidad intelectual, inteligencia emocional, optimismo, pesimismo.

\begin{abstract}
The adaptive use of emotions in workers who practice their profession with people with intellectual disability is crucial to do their job. This research analyses the relationship between emotional intelligence and optimism vs. pessimism. The sample consists of 59 subjects $(n=59)$, with an average age of 38.59 years $( \pm 10,359)$, and who belong to different assistance centers. The following instruments are used: Trait Meta-Mood Scale-24 (TMMS-24), Emotional Quotient Inventory (EQi-C), and Life Orientation Test Revised (LOT-R). The use of the Omega coefficient is noteworthy because it is an adequate measure of reliability if there is no principle of equivalence. Evidence shows the relationship between some of the dimensions of the instruments considered $(p<.05)$ and there are significant statistical differences between the emotional intelligence dimensions and the sociodemographic variable gender $(p<.05)$. The regression analysis confirms the association between emotional intelligence (reparation) with optimism and emotional intelligence (stress management) and pessimism.
\end{abstract}

\section{Keywords}

Intellectual disability, emotional intelligence, optimism, pessimism.

\author{
Óscar Gavín-Chocano \\ <oscar_gavin@yahoo.es> \\ Universidad de Jaén. España
}

David Molero López-Barajas $<$ dmolero@ujaen.es>

Universidad de Jaén. España

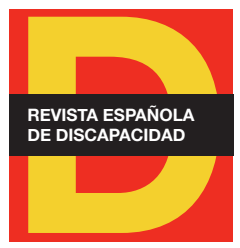

Para citar:

Gavín-Chocano, O. y Molero, D. (2020). "Relación entre inteligencia emocional y optimismo vs. pesimismo en trabajadores de centros para personas con discapacidad intelectual". Revista Española de Discapacidad, 8(I), pp. 129-144.

Doi: <https://doi.org/10.5569/23405104.08.01.07>

Fecha de recepción: 24-10-2019 Fecha de aceptación: 21-01-2020

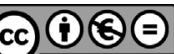




\section{Introducción}

A finales del siglo XX, ligado al desarrollo de nuevas políticas sociales para la ciudadanía y la mejora de su calidad de vida, denominado Estado del Bienestar, también se comenzó a potenciar el concepto de inteligencia emocional (en adelante IE), dando respuesta así a la necesaria fusión entre emoción y razón. Sin duda, un aspecto fundamental para el desarrollo personal en diferentes ámbitos, entre ellos los relacionados con la actividad laboral (Mayer et al., 2016). Si bien la conceptualización del constructo (Salovey y Mayer, 1990) y las diferentes estrategias en su inicio centraron su contenido en la configuración de modelos teóricos e instrumentos para su evaluación (Mayer et al., 2001), en la actualidad se hace imprescindible la relación e influencia de la IE con otras variables capaces de mostrar la verdadera potencialidad como elemento regulador del componente emocional (Pulido-Acosta y Herrera-Clavero, 2018).

En el ámbito de profesionales que atienden a personas con discapacidad intelectual, el desarrollo de nuevas estrategias resultó fundamental. La gestión emocional es determinante, pues las circunstancias de la actividad laboral, la implicación emocional y el contacto directo con personas que, por lo general, se encuentran en situación de vulnerabilidad, puede favorecer el desgaste y deterioro en el desempeño de la labor realizada (Extremera et al., 2005; Vizoso-Gómez y Arias-Gundín, 2018). Diferentes investigaciones han demostrado cómo la IE favorece estados de ánimo optimistas, siendo un buen predictor del funcionamiento personal y profesional de trabajadores asistenciales (Gómez-Ortiz et al., 2016; Morales-Rodríguez, 2017).

\subsection{Inteligencia emocional}

Desde su conceptualización hasta hoy, como habilidad en la gestión y uso adaptativo de las emociones propias, comprendiendo las de los demás, utilizando esa información de manera eficaz (Cazalla-Luna y Molero, 2018; Fernández-Berrocal et al., 2018), regulando el comportamiento de acuerdo a la situación (Cejudo et al, 2016; Zych et al.,2016), se han desarrollado diferentes modelos teóricos, donde podemos destacar dos, por su importancia y características generales: el modelo habilidad y el modelo rasgo. El primero centra su contenido en las destrezas que utiliza el individuo para gestionar la información recibida a través de las emociones (Mayer et al., 2001). Respecto a su evaluación, este constructo debe ser medido con pruebas de rendimiento máximo, siendo uno de los instrumentos más utilizados el Trait Meta-Mood Scale -TMMS- (Salovey et al., 1995). El modelo rasgo, por otro lado, conceptualiza la IE como la combinación de habilidades mentales y rasgos de la personalidad (Mayer et al., 2008), debiendo ser evaluado con pruebas de rendimiento típico, siendo la más utilizada la Bar-On Emotional Quotient Inventory -EQi- (Bar-On, 2006).

Si bien dichos modelos comparten aspectos básicos en su origen, como componente regulador de las emociones (Veloso-Besio et al., 2013), también se diferencian en la forma de analizarlos, centrado el modelo habilidad en la interacción entre pensamiento y emoción, y el modelo rasgo en el conjunto de rasgos estables de la personalidad, motivaciones, competencias socioemocionales y habilidades cognitivas (Bar-On y Parker, 2000), configurando una visión más amplia sobre el modelo restringido de habilidad (FernándezBerrocal y Extremera, 2006).

Desde este marco, este estudio centra su contenido en el análisis de sendas tendencias para profesionales que atienden a personas con discapacidad intelectual entendiendo que, a partir de estudios realizados con 
anterioridad, el modo en cómo se conceptualice la IE está directamente relacionado con la fundamentación del constructo en diferentes aspectos de la vida (López-Fernández, 2015) y su evolución a lo largo del tiempo (Suriá, 2017). En este sentido, numerosos trabajos relacionan altos niveles de IE con estados de ánimo positivos y un mayor ajuste psicológico en profesionales que ejercen su actividad laboral (Cejudo et al., 2018) y disposición al optimismo (Augusto-Landa et al., 2011). Otros estudios sostienen que bajos niveles de IE se relacionan con desgaste laboral y sintomatología depresiva y pesimista (Cazalla-Luna y Molero, 2016; Extremera et al., 2005; Nespereira-Campuzano y Vázquez-Campo, 2017). Igualmente se relaciona la IE con un mayor rendimiento laboral en profesionales que ejercen su profesión en colectivos desfavorecidos, constatando mayores puntuaciones tanto en IE habilidad como rasgo (O'Boyle et al., 2011).

\subsection{Disposición al optimismo vs. pesimismo}

Otros conceptos que han ido adquiriendo relevancia en diferentes ámbitos relacionados con el bienestar y la salud emocional del individuo, son el optimismo vs. pesimismo (Knowlden et al., 2016; Vizoso-Gómez y Arias-Gundín, 2018), relacionados con la percepción del individuo sobre eventos futuros y siendo determinantes cuando la persona se enfrenta a situaciones estresantes o acciones que ponen en riesgo su bienes$\operatorname{tar}$ (Moya-Smith, 2018).

Desde una perspectiva teórica, tanto optimismo como pesimismo han sido analizados a partir de enfoques diferentes (Ortiz et al., 2016). Por un lado, el modelo teórico optimismo disposicional considera los acontecimientos positivos fruto de acciones propias, haciendo extensible su recorrido en un futuro inmediato, en todas las áreas vitales. Por el contrario, el modelo atributivo optimista (Gillham et al., 2002), percibe los acontecimientos negativos de forma externa, diferenciada, aislada y no prolongable a otras áreas vitales (Peterson y Seligman, 1984; Remor et al., 2006). La diferencia en ambos modelos reside en las atribuciones sobre eventos pasados y futuros, positivos y negativos, asumiendo en ambos rasgos de la personalidad y estrategias adaptativas (Cazalla-Luna y Molero, 2016; Xia et al., 2016).

\section{Figura 1. Estilo atributivo optimismo vs. pesimismo}

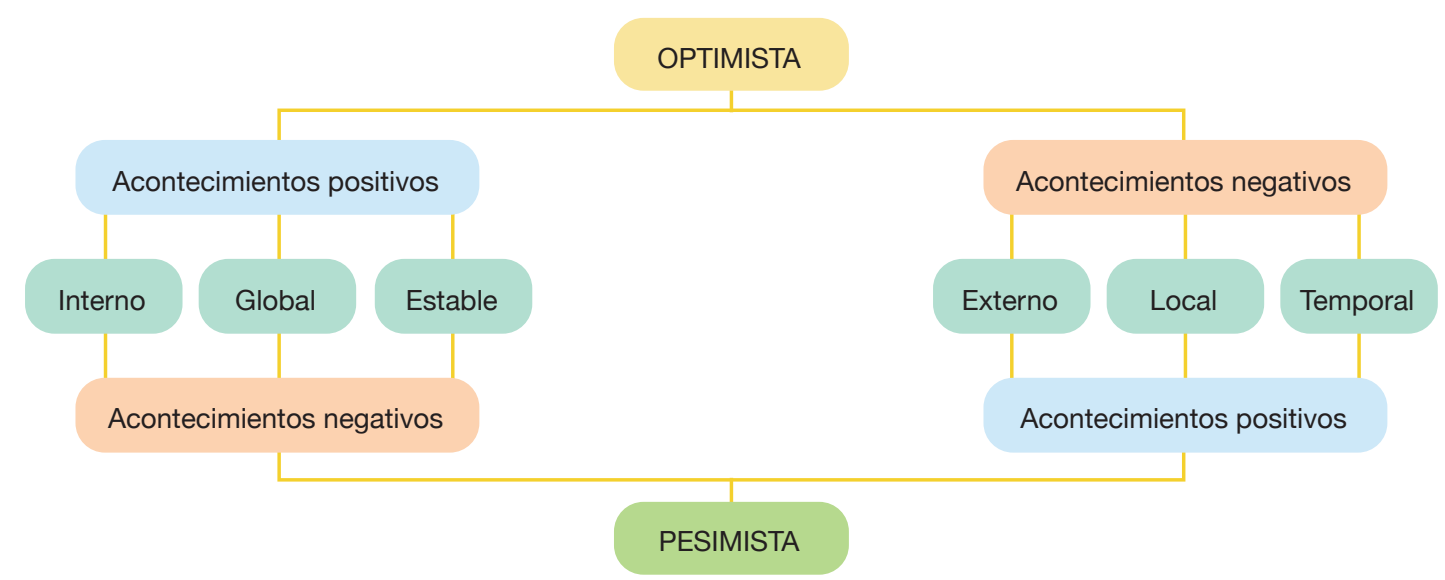

Fuente: elaboración propia. 
Este estudio parte del segundo modelo, entendiendo que optimismo vs. pesimismo son simples interpretaciones de la realidad cotidiana (Sanin y Salanova-Soria, 2018), pudiendo diferenciar ambas variables. Si bien la persona pesimista, al percibir efectos dañinos, evitará los diferentes retos adversos (Blas, 2016), repercutiendo de forma negativa en el logro de objetivos (Carver y Scheier, 2014); la persona optimista, en cambio, realizará un mayor esfuerzo para conseguir sus metas, utilizando estrategias de afrontamiento para situaciones desequilibrantes (Cazalla-Luna y Molero, 2018).

Las evidencias disponibles, señalan que optimismo vs. pesimismo se asocian con una mayor o menor disposición para afrontar situaciones estresantes (Carver y Scheier, 2014). Son muchos los estudios que señalan la disposición al optimismo como reductor de estresores laborales (Villardefrancos et al., 2012). De la misma forma, las personas optimistas presentan mayores niveles de IE en el desempeño de su trabajo (Extremera et al., 2005), siendo más eficaces (Feldman y Kubota, 2015), presentando niveles más bajos de ansiedad (Pino et al., 2016).

Constatando la influencia de la IE sobre las variables optimismo vs. pesimismo en el ámbito de trabajadores que atienden a personas con discapacidad intelectual, aún existen pocos estudios que aborden la relación de estas variables para explicar el efecto de la dimensión emocional en el desarrollo de la actividad laboral (Llorent y Ruiz-Calzado, 2016). Es decir, las habilidades emocionales del profesional para gestionar situaciones complejas pueden mejorar la capacidad de afrontamiento y aumentar su optimismo (Montero et al., 2014; Huerta-Ramírez y Rivera-Heredia, 2017). Se fundamenta que la adquisición de competencias emociones y una actitud optimista resultan determinantes para un mejor rendimiento y la superación de las dificultades cotidianas (Cejudo et al., 2018).

A partir de estas consideraciones, en el presente trabajo se plantean tres objetivos con carácter general. En primer lugar, analizar la existencia de correlaciones significativas entre las dimensiones de los instrumentos de IE (EQi-C y TMMS-24), a partir de los modelos habilidad y rasgo, y disposición al optimismo vs. pesimismo (LOT-R). En segundo lugar, determinar la existencia de diferencias significativas entre cada una de las variables de los instrumentos (TMMS-24, EQi-C y LOT-R) y las variables sociodemográficas (género y edad). Por último, predecir qué variables de la IE son las que explican el optimismo y pesimismo de los sujetos a estudio.

Cabe señalar los ajustes y fiabilidad de las pruebas a través de alfa de Cronbach y coeficiente Omega (McDonald, 1999), también denominado Rho de Jöreskog (Stone et al., 2015), al trabajar la suma ponderada de cada variable y salvar las limitaciones que podrían afectar a la varianza (Domínguez-Lara y Merino-Soto, 2015).

\section{Método}

El diseño del presente estudio desarrolla estrategias de la investigación asociativa y descriptiva correlacional, empleando medidas de carácter transversal, comparativas y de predicción. (Ato et al., 2013). 


\subsection{Participantes}

Para la realización de este estudio se seleccionó una muestra de diferentes profesionales que ejercen su actividad profesional con personas con discapacidad intelectual en la provincia de Jaén (España): Agencia de Servicios Sociales y Dependencia de la provincia de Jaén, Asociación de Personas con Discapacidad Intelectual AINPER de Linares, Asociación de Familiares y Amigos de Personas con Discapacidad Intelectual AFAMP de Bailén, Asociación de Personas con Discapacidad de Jódar, Centro Ocupacional de Discapacitados de Bailén, Centro de Atención a Minusválidos Psíquicos CAMP de Linares, I.E.S. Medina Rivilla de Bailén. Por sexo, los participantes $(n=59)$ son 48 mujeres, que suponen el 81,40\% y 11 hombres $18,60 \%$. El rango de edad oscila entre 19 y 57 años, con una media de $38.59( \pm 10.359)$. Hemos empleado un muestreo no probabilístico de tipo causal o accidental.

Se empleó un muestreo no probabilístico de tipo casual o por accesibilidad (Latorre et al., 2003), considerando fundamental contactar con un número determinado de centros y asociaciones que atienden a personas con discapacidad intelectual y sus trabajadores, con el fin de alcanzar una muestra representativa considerable. La muestra resultó de aquellos trabajadores que accedieron voluntariamente a responder los diferentes cuestionarios.

\subsection{Instrumentos}

Para recoger información relevante, se han incluido dos variables sociodemográficas relativas a género y edad, con el propósito de obtener evidencias de la muestra y analizar la existencia de diferencias significativas en función de éstas. A continuación, se detallan las características y propiedades psicométricas de los instrumentos de evaluación considerados:

Trait Meta-Mood Scale-24-. Para evaluar la IE se utilizó la escala Trait Meta-Mood Scale-24 (TMMS-24), de Fernández-Berrocal et al. (2004), versión original de Salovey et al. (1995), que engloba tres dimensiones (atención, claridad y reparación emocional). Esta herramienta ha sido utilizada en multitud de contextos de investigación en Ciencias Sociales (Tolegenova et al., 2014) y de las Ciencias de la Salud (Aradilla-Herrero et al., 2014; Lara et al., 2014; Munkholm et al., 2012). En la muestra a estudio la fiabilidad de las puntuaciones para cada subescala es de $\mathrm{a}=.89$ para atención, $\mathrm{a}=.85$ en claridad y $\mathrm{a}=.83$ para reparación, respectivamente. Igualmente se informa del coeficiente Omega $(\omega)$, al considerar la suma ponderada de las variables con el propósito de reflejar el verdadero nivel de fiabilidad. Estas son: atención $\omega=.80$, claridad $\omega=.86$ y reparación $\omega=.82$.

Emotional Quotient Inventory. Para evaluar la IE se utilizó la Emotional Quotient Inventory -EQi-C- (LópezZafra et al., 2014). Es una adaptación al español (versión corta) del EQ-i (Bar-On, 1997) y su versión para jóvenes y adolescentes EQ-i: YV (Bar-On y Parker, 2000). Ofrece información relativa a competencias emocionales y su relación con otras variables, contando con cuatro factores (interpersonal, adaptabilidad, manejo del estrés e intrapersonal) para el estudio de la IE, siendo los valores de la consistencia interna informados por sus autores de $a=.78, a=.75, a=.73$ y $a=.70$ para cada sub-escala. En nuestra muestra la fiabilidad (a) de las puntuaciones para cada sub-escala del EQi-C es de $a=.74, a=0.80, a=.82$ y $a=.67$, siendo la fiabilidad Omega $(\omega)$ de $\omega=.82, \omega=.69, \omega=.83$ y $\omega=.66$, respectivamente. 
Life Orientation Test Revised (LOT-R). Para evaluar la disposición al optimismo vs. pesimismo se utilizó la versión en español del Life Orientation Test Revised-LOT-R- (Remor et al., 2006), escala compuesta por diez ítems que miden el grado de optimismo (siempre estoy optimista sobre mi futuro) o pesimismo (nunca espero que las cosas salgan a mi manera) de los sujetos. El formato de respuesta responde a una escala de tipo Likert de cinco puntos. Cuenta con 10 ítems, de los cuales 3 evalúan el grado de positivismo del sujeto, y los 3 restantes el grado de pesimismo del mismo, siendo el resto de relleno en el cuestionario. Los participantes señalarán el grado de acuerdo o desacuerdo en cada afirmación, desde 0 (muy en desacuerdo), hasta 4 (muy de acuerdo). Se estima que, a mayor valoración, mayor optimismo disposicional (Feldman y Kubota, 2015). La consistencia interna fiabilidad (a) informada por sus autores es de $a=.78$; siendo la fiabilidad de las puntuaciones en nuestro estudio de $a=.56$ para optimismo y $a=.82$ para pesimismo y la fiabilidad Omega $(\omega)$ de $\omega=.54$ para optimismo y $\omega=.78$ para pesimismo, respectivamente.

\subsection{Procedimiento}

El estudio se llevó a cabo obteniendo consentimiento informado de cada participante. Los sujetos fueron debidamente informados del proceso a seguir, confidencialidad y anonimato de las informaciones recogidas. Cada una de las pruebas se realizó de forma individual. Toda la información relativa al propósito del estudio fue remitida a los responsables de los centros, junto a una carta de presentación informando de los objetivos generales del estudio. Se entregaron los cuestionarios siguiendo los criterios establecidos, siendo el orden siguiente; en primer lugar, el Trait Meta-Mood Scale-24 (TMMS-24), seguido por el Emotional Quotient Inventory (EQi-C), y a continuación, el Life Orientation Test Revised (LOT-R). A pesar de optar por esta secuencia, este orden no varía el resultado previsto.

\subsection{Análisis estadístico}

A partir de los datos recogidos, se obtuvieron los estadísticos descriptivos (medias y desviaciones típicas), analizando a priori la fiabilidad de las puntuaciones de cada instrumento, consistencia interna, alfa de Cronbach y coeficiente Omega, también conocido como Rho de Jöreskog (Stone et al., 2015) y la correlación entre las puntuaciones resultantes en cada una de las dimensiones. A continuación, se realizó un análisis de diferencias de medias en función del género (prueba $t$ de Student de diferencia de medias para pruebas independientes); para la variable edad (expresada en tres intervalos) se empleó un ANOVA. Se verificaron en todos los casos los supuestos de independencia de datos, normalidad y bondad de ajuste a través de la prueba de Kolmogorov-Smirnov, y también el de aditividad en el ANOVA (Gil-Pascual, 2015), además de comunicar el tamaño del efecto. Finalmente, para explorar el valor predictivo de las variables IE sobre el optimismo vs. pesimismo se realizaron dos estudios de regresión paso a paso diferenciando ambas variables (optimismo y pesimismo), respectivamente. En todos los casos se empleó un nivel de confianza del $95 \%$ (significación $p<.05$ ), empleando el programa SPSS 22.0 (IBM, Chicago, IL) para obtener los resultados de las pruebas indicadas con anterioridad. 


\section{Resultados}

Los resultados obtenidos se presentan en función de los objetivos planteados previamente, dando continuidad a cada una de las respuestas de cada uno de ellos a continuación.

\subsection{Relación entre optimismo, pesimismo e inteligencia emocional}

En primer lugar, presentamos en la Tabla 1, la matriz de correlaciones, estadísticos descriptivos y la fiabilidad de las puntuaciones (ya señaladas en el apartado anterior).

Tabla 1. Consistencia interna, medias, desviación típica y correlaciones ( $r$ de Pearson) entre las variables optimismo, pesimismo e inteligencia emocional

\begin{tabular}{|l|c|c|c|c|c|c|c|c|c|c|c|c|}
\hline Variable & $\mathbf{a}$ & $\omega$ & $\mathbf{M}(\mathbf{D T})$ & OPT & $\mathbf{P E S}$ & $\mathbf{A T}$ & $\mathbf{C L}$ & $\mathbf{R E}$ & INTE & ADAP & EST & INTR \\
\hline OPT & .56 & .54 & $10.08( \pm 2.18)$ & - & $.26^{\star}$ & .40 & .16 & $.49^{\star}$ & .06 & .14 & -.09 & .14 \\
\hline
\end{tabular}

\begin{tabular}{|l|l|l|l|l|l|l|l|l|l|l|l|l|}
\hline PES & .82 & .78 & $12.54( \pm 2.47)$ & & - & .15 & .14 & $.25^{\star}$ & .02 & -.13 & $-.49^{\star}$ & $.48^{\star \star}$ \\
\hline
\end{tabular}

\begin{tabular}{|l|c|c|c|c|c|c|c|c|c|c|c|c|}
\hline AT & .89 & .80 & $24.19( \pm 5.95)$ & & & - & -.10 & -.14 & .14 & -.07 & .14 & -.18 \\
\hline CL & .85 & .86 & $27.73( \pm 5.27)$ & & & & - & $.43^{* *}$ & $.28^{\star}$ & $.41^{* *}$ & -.24 & .22 \\
\hline RE & .83 & .82 & $28.31( \pm 5.58)$ & & & & & - & $.32^{\star}$ & $.40^{*}$ & -.15 & .15 \\
\hline & & & & & & & & & & & & \\
\hline INTE & .74 & .82 & $28.08( \pm 4.02)$ & & & & & & - & $.54^{* *}$ & $-.38^{* \star}$ & .18 \\
\hline ADAP & .80 & .69 & $18.37( \pm 3.65)$ & & & & & & & - & -.05 & -.08 \\
\hline EST & .82 & .83 & $15.36( \pm 5.72)$ & & & & & & & & - & $-.68^{\star *}$ \\
\hline INTR & .67 & .66 & $32.05( \pm 5.88)$ & & & & & & & & & - \\
\hline
\end{tabular}

Nota: (1) Media=M, Desviación típica=DT, Optimismo=OPT, Pesimismo=PES, Inteligencia emocional Atención=AT, Claridad=CL, Reparación=RE, Inteligencia emocional Interpersonal=INTE, Adaptabilidad=ADAP, Manejo del estrés=EST, Intrapersonal=INTR. $(2){ }^{*}=p<.05 ;{ }^{* *}=p<.01$.

Fuente: elaboración propia.

Los resultados indican que existe relación positiva estadísticamente significativa entre las variables optimismo y pesimismo $(r=.26 ; p<.05)$, también entre optimismo y la variable de IE reparación $(r=.49 ; p<.05)$. Igualmente existe relación significativa entre las variables pesimismo e IE reparación $(r=.25 ; p<.05)$ en el instrumento TMMS-24. Respecto a la relación entre la variable pesimismo, encontramos diferencias significativas con la IE manejo del estrés de manera inversa $(r=-.49 ; p<.05)$ e IE intrapersonal de forma positiva $(r=.48 ; p<.01)$, en el instrumento EQi-C. Respecto a la asociación entre las variables de IE TMMS-24 y EQi-C, se constata la relación positiva de la IE claridad con la IE reparación ( $r=.43 ; p<.01)$, IE interpersonal $(r=.28$; $p<.05)$ e IE adaptabilidad $(r=.41 ; p<.01)$. Por otro lado, encontramos diferencias significativas entre IE repa- 
ración e interpersonal $(r=.32 ; p<.05)$ y adaptabilidad $(r=.40 ; p<.05)$. También entre IE interpersonal y adaptabilidad $(r=.54 ; p<.01)$ y manejo del estrés de manera inversa $(r=-.38 ; p<.01)$. Por último, también se constata la relación significativa negativa entre las sub-escalas de IE manejo del estrés e intrapersonal $(r=-.68 ; p<.01)$.

\subsection{Diferencias en función de las variables sociodemográficas}

Para explorar las diferencias en relación al género se ha empleado la prueba $t$ de Student para muestras independientes (véase Tabla 2). Los resultados indican que solo existen diferencias significativas entre dos de las dimensiones de IE del instrumento EQi-C, manejo del estrés $\left(t_{56}=3.60 ; p<.05\right)$, obteniendo una puntuación más alta los hombres que las mujeres, y de forma inversa, la sub-escala intrapersonal $\left(t_{56}=-2.46\right.$; $p<.05)$, siendo la valoración más elevada en mujeres.

\begin{tabular}{l}
\hline Tabla 2. Diferencias de medias en función del género (Prueba t de Student) \\
\begin{tabular}{|l|c|c|c|c|c|}
\hline Variables & $\begin{array}{c}\text { Hombres }(\mathbf{n}=\mathbf{1 1}) \\
\boldsymbol{M}(\boldsymbol{D T})\end{array}$ & $\begin{array}{c}\text { Mujeres }(\mathbf{n}=\mathbf{4 8}) \\
\boldsymbol{M}(\boldsymbol{D} \boldsymbol{T})\end{array}$ & $\boldsymbol{t}(\mathbf{5 6})$ & $\boldsymbol{p}$ & Efecto (d) \\
\hline & & & & & \\
OPT & $11.09( \pm 1.70)$ & $9.85( \pm 2.23)$ & 1.723 & .429 & .625 \\
PES & $12.36( \pm 2.97)$ & $12.58( \pm 2.37)$ & -.264 & .732 & .081 \\
AT & $26.73( \pm 4.56)$ & $23.60( \pm 6.11)$ & 1.590 & .231 & .580 \\
CL & $26.91( \pm 4.59)$ & $27.92( \pm 5.44)$ & -.568 & .499 & .200 \\
RE & $29.73( \pm 3.25)$ & $27.98( \pm 5.97)$ & .936 & .108 & .360 \\
INTER & $26.09( \pm 3.53)$ & $28.54( \pm 4.02)$ & -1.861 & .923 & .647 \\
ADAP & $18.27( \pm 3.95)$ & $18.40( \pm 363)$ & -.100 & .829 & .034 \\
EST & $20.45( \pm 8.28)$ & $14.19( \pm 4.27)$ & 3.600 & $.001^{*}$ & .950 \\
INTRA & $28.27( \pm 7.51)$ & $32.92( \pm 5.15)$ & -2.463 & $.050^{*}$ & .722 \\
\hline
\end{tabular}
\end{tabular}

Nota: (1) Optimismo=OPT, Pesimismo=PES, Inteligencia emocional Atención=AT, Claridad=CL, Reparación=RE, Inteligencia emocional Interpersonal=INT, Adaptabilidad=ADAP, Manejo del estrés=EST, Intrapersonal=INTRA. (2) ${ }^{*}=p<.05 ;{ }^{* *}=p<.01$. (3) El tamaño del efecto estadístico está expresado con el valor de Cohen.

Fuente: elaboración propia.

En el resto de variables de IE EQi-C no se encontraron diferencias significativas en relación al género $\left(t_{56}<2.0\right.$; $p>.05 \mathrm{~ns})$. Igualmente, no existen diferencias significativas ni en optimismo $\left(t_{56}<2.0 ; p>.05 \mathrm{~ns}\right)$, ni pesimismo $\left(t_{56}<2.0 ; p>.05 \mathrm{~ns}\right)$. Tampoco en ninguna de las dimensiones del TMMS-24 hemos encontrado diferencias significativas en relación al género $\left(t_{56}<2.0 ; p>.05 \mathrm{~ns}\right)$. El tamaño del efecto es pequeño en optimismo, IE claridad y adaptabilidad $(d<2)$, y alto en la sub-escala de IE manejo del estrés $(d<8)$. El resto de variables tienen un efecto mediano siguiendo los criterios clásicos de Cohen (1988).

Para analizar las diferencias en función de la edad se determinaron tres intervalos (19-31 años, 26-39 años y +45 años) realizándose un ANOVA (véase Tabla 3). En las dimensiones optimismo $\left(F_{(2,56)}=.499 ; p>.05\right.$ ns) y pesimismo $\left(F_{(2,56)}=1.007 ; p>.05 \mathrm{~ns}\right)$, no se encontraron diferencias significativas en relación a la edad. En las dimensiones de IE de TMMS-24 tampoco se encontraron diferencias significativas $\left(F_{(2,56)}<2.0 ; p>.05 \mathrm{~ns}\right)$. 


\begin{tabular}{|c|c|c|c|c|c|c|}
\hline Variable & $\begin{array}{c}\text { 19-31 años } \\
M(D T)\end{array}$ & $\begin{array}{c}\text { 32-44 años } \\
M \text { (DT) }\end{array}$ & $\begin{array}{c}\text { 45-57 años } \\
M(D T)\end{array}$ & $F(2,56)$ & $p$ & $\begin{array}{c}\text { Efecto } \\
\eta^{2}\end{array}$ \\
\hline OPT & $10.00( \pm 1.77)$ & $9.81( \pm 2.42)$ & $10.50( \pm 2.35)$ & .499 & .610 & .017 \\
\hline PES & $12.65( \pm 2.45)$ & $13.00( \pm 2.38)$ & $11.89( \pm 2.58)$ & 1.007 & .372 & .035 \\
\hline AT & $23.75( \pm 4.85)$ & $25.24( \pm 6.35)$ & $23.44( \pm 6.69)$ & .513 & .602 & .018 \\
\hline$C L$ & $27.45( \pm 5.60)$ & $26.43( \pm 5.25)$ & $29.56( \pm 4.63)$ & 1.796 & .175 & .060 \\
\hline RE & $28.25( \pm 5.76)$ & $27.05( \pm 6.81)$ & $29.83( \pm 3.18)$ & 1.217 & .304 & .042 \\
\hline INTER & $29.10( \pm 4.24)$ & $27.65( \pm 3.46)$ & $27.44( \pm 4.36)$ & .978 & .383 & .034 \\
\hline ADAP & $18.90( \pm 3.82)$ & $17.10( \pm 3.49)$ & $19.28( \pm 3.42)$ & 2.119 & .130 & .070 \\
\hline EST & $13.80( \pm 4.84)$ & $15.00( \pm 4.31)$ & $17.50( \pm 7.46)$ & 2.125 & .129 & .071 \\
\hline INTRA & $32.45( \pm 5.37)$ & $32.86( \pm 5.25)$ & $30.67( \pm 7.08)$ & .735 & .484 & .026 \\
\hline
\end{tabular}

Nota: (1) Optimismo=OPT, Pesimismo=PES, Inteligencia emocional Atención=AT, Claridad=CL, Reparación=RE, Inteligencia emocional Interpersonal=INTER, Adaptabilidad=ADAP, Manejo del estrés=EST, Intrapersonal=INTRA. (2) ${ }^{*}=p<.05 ;{ }^{* *}=p<.01$. (3) El tamaño del efecto estadístico está expresado con el valor Eta cuadrado $\left(\eta^{2}\right)$.

Fuente: elaboración propia.

En las variables IE EQi-C, tampoco se han encontrado diferencias significativas con la edad $\left(F_{(2,56)}<2.0\right.$; p>.05 ns). La prueba HSD de Tukey informa de diferencias significativas entre los participantes de entre 3244 años y los de mayores de 45 años, a favor de los de más edad, siendo el tamaño del efecto pequeño en todos los casos $\left(\eta^{2}\right)$.

\subsection{Estudio de regresión}

Con el propósito de analizar las variables que predicen optimismo vs. pesimismo (LOT-R) consideradas como variables criterio, se han realizado dos análisis de regresión lineal a través del método pasos sucesivos. Se establecieron como variables predictoras cada una de las dimensiones de la IE del TMMS-24 (atención, claridad y reparación), EQi-C (interpersonal, adaptabilidad, manejo del estrés e intrapersonal) respectivamente asegurando, en primera instancia, que no existiese ningún problema de multicolinealidad (valores de tolerancia <.20; FIV >4.00).

El resumen del modelo para la variable optimismo (véase Tabla 4), indica que sólo queda incluida la dimensión de IE reparación (RE) del instrumento TMMS-24 quedando excluidas el resto de las sub-escalas (atención -A- y claridad -C-) y las cuatro dimensiones de IE del instrumento EQi-C (interpersonal -INT-, adaptabilidad -ADAP-, manejo del estrés-EST- e intrapersonal -INTR-).

La dimensión incluida en la regresión explica un $24,1 \%$ de la varianza $\left(\mathrm{R}=.491 ; R^{2}\right.$ corregida=.241; $F=18.137$ $p<.05)$, existiendo un valor de $t$ significativo, siendo la mejor variable predictora del optimismo. 


\begin{tabular}{l}
\hline Tabla 4. Análisis de regresión lineal (pasos sucesivos), variable criterio: optimismo \\
\begin{tabular}{|l|c|c|c|c|c|c|c|}
\hline $\begin{array}{l}\text { Variable } \\
\text { criterio }\end{array}$ & $\boldsymbol{R}$ & $\boldsymbol{R}^{2}$ & $\begin{array}{c}\boldsymbol{R}^{2} \\
\text { Corregida }\end{array}$ & $\boldsymbol{F}$ & $\begin{array}{c}\text { Variables } \\
\text { predictoras }\end{array}$ & Beta & $\boldsymbol{t}$ \\
\hline & .491 & .241 & .228 & 18.137 & & .192 & $4.259^{* *}$ \\
\hline
\end{tabular}
\end{tabular}

Nota: (1) Optimismo=OPT, Inteligencia emocional Atención=A, Claridad=CL, Reparación=RE, Inteligencia emocional Interpersonal=INTER, Adaptabilidad=ADAP, Manejo del estrés=EST, Intrapersonal=INTRA. (2) ${ }^{* *}=p<.01$.

Fuente: elaboración propia.

De igual forma, el resumen del modelo para la variable pesimismo (véase Tabla 5), indica que sólo queda incluida la dimensión de IE manejo del estrés (EST) del instrumento EQi-C quedando excluidas el resto de las sub-escalas (interpersonal -INT-, adaptabilidad -ADAP-, e intrapersonal -INTRA-) y las tres dimensiones de IE del instrumento TMMS-24 (atención -AT-, claridad -CL- y reparación -RE-).

Tabla 5. Análisis de regresión lineal (pasos sucesivos), variable criterio: pesimismo

\begin{tabular}{|c|c|c|c|c|c|c|c|}
\hline $\begin{array}{l}\text { Variable } \\
\text { criterio }\end{array}$ & $R$ & $R^{2}$ & $\begin{array}{c}R^{2} \\
\text { Corregida }\end{array}$ & $\boldsymbol{F}$ & $\begin{array}{c}\text { Variables } \\
\text { predictoras }\end{array}$ & Beta & $t$ \\
\hline \multirow{2}{*}{ Pesimismo } & .494 & .244 & .231 & 18.418 & & & \\
\hline & & & & & EST & .214 & $4.292^{\star \star}$ \\
\hline
\end{tabular}

Nota: (1) Pesimismo=PES, Inteligencia emocional Atención=AT, Claridad=CL, Reparación=RE, Inteligencia emocional Interpersonal=INTER, Adaptabilidad=ADAP, Manejo del estrés=EST, Intrapersonal=INTRA. (2) ${ }^{* *}=p<.01$.

Fuente: elaboración propia.

La dimensión incluida en la regresión explica un $24,4 \%$ de la varianza $\left(\mathrm{R}=.494 ; R^{2}\right.$ corregida=.244; $F=18.418$ $p<.05)$, existiendo un valor de $t$ significativo, siendo la mejor variable predictora del pesimismo.

\section{Discusión}

En el presente trabajo se analizó la relación entre las dimensiones de IE para el modelo habilidad (atención, claridad y reparación), las dimensiones de IE para el modelo rasgo (interpersonal, adaptabilidad, manejo del estrés e intrapersonal) y optimismo vs. pesimismo, diferenciando ambos conceptos, en profesionales que ejercen su actividad laboral con personas con discapacidad intelectual.

A priori se verificó la fiabilidad (consistencia interna) de cada uno de los instrumentos a través del cálculo alfa de Cronbach, por ser el más utilizado, y posteriormente el coeficiente Omega, considerando que las 
limitaciones del primero podrían afectar a la proporción de la varianza (Domínguez-Lara y Merino-Soto, 2015). El coeficiente Omega trabaja con la suma ponderada de las variables, haciendo más fiable su cálculo. En relación con el primer objetivo, los resultados encontrados evidenciaron diferencias significativas entre las variables optimismo vs. pesimismo. Se constata la validez psicométrica del instrumento LOT-R para medir las dos variables de forma independiente (Xia et al., 2016). Estos datos replican otros estudios que sostienen que las variables optimismo vs. pesimismo no son términos antagónicos, sino extremos del mismo constructo con efecto diferente (Sanin y Salanova-Soria, 2018). De igual forma, existe relación significativa entre IE (reparación) y optimismo vs. pesimismo, coincidiendo con otros trabajos que mantienen que las personas que son capaces de sobreponerse o no, a situaciones adversas, alcanzan un mayor o menor nivel de bienestar psicológico (Cejudo et al.,2016). Además, los resultados muestran la relación entre IE (manejo del estrés intrapersonal) y pesimismo. Los efectos del estrés psicológico tienen consecuencias diferentes en función del factor moderador, más optimista o pesimista (Carver y Scheier, 2014; Ortiz et al., 2016). Esta distancia queda justificada en relación con el esfuerzo que realizan las personas por lograr sus objetivos cuando perciben que son alcanzables (Nespereira-Campuzano y Vázquez-Campo, 2017).

Al analizar la concordancia entre IE en el modelo habilidad y rasgo, se constata la relación entre alguna de las variables del instrumento TMMS-24 (claridad y reparación) y las variables del instrumento EQi-C (interpersonal, adaptabilidad y manejo del estrés), coincidimos con los hallazgos obtenidos por otros autores que sostienen que la variable atención no correlaciona con ninguna sub-escala de IE (Cazalla-Luna y Molero, 2016; Extremera et al, 2005). Es necesario señalar que las personas que muestran más claridad respecto a la gestión de sus emociones son capaces de adaptarse y manejar situaciones estresantes y, por tanto, alcanzar un mayor bienestar (Cejudo et al., 2016).

En cuanto al segundo objetivo, determinar la relación en optimismo vs. pesimismo con la variable sociodemográfica género, no se encuentran diferencias significativas, situando a los hombres con puntuaciones más altas en optimismo y a las mujeres en pesimismo. Estos resultados coinciden con otros autores que mantienen mayores índices de optimismo en mujeres en el entorno laboral (Giménez, 2005). Puede ser que los resultados vengan condicionados por el mayor número de mujeres de la muestra, lo cual dificultaría la validez de la evidencia resultante (Llorent y Ruiz-Calzado, 2016).

Por otra parte, cabe destacar la relación de la IE en el instrumento EQi-C con género, siendo las mujeres las que presentaron mayores niveles de IE (intrapersonal). Datos que coinciden con otros estudios que sostienen que las mujeres obtienen valores más altos en aspectos relacionados con la comprensión y expresión de las emociones (Fernández-Berrocal et al., 2018; López-Zafra et al., 2014). Sin embargo, los hombres obtienen puntuaciones más elevadas en IE (manejo del estrés). Estos datos corroboran otros estudios que evidencian una mayor regulación emocional ante situaciones adversas por parte de los hombres (CazallaLuna y Molero, 2018).

Respecto a la relación entre IE y la variable sociodemográfica edad, se constata no encontrar diferencias significativas, siendo los más optimistas los sujetos de más edad, circunstancia paralela en el pesimismo. El contacto directo en profesiones asistenciales influye de manera significativa en la pérdida de perspectiva y despersonalización dentro del ámbito laboral (Llorent y Ruiz-Calzado, 2016). A medida que aumenta la edad, este estado aumenta progresivamente (Pulido-Acosta y Herrera-Clavero, 2018). 
Tampoco existe relación en las variables de IE habilidad y rasgo con la edad, siendo los sujetos de más edad los que puntúan más alto. Puede ser que los profesionales de más de 45 años hayan adquirido las competencias y herramientas necesarias para un buen desarrollo en su actividad profesional. Sin embargo, otros estudios contradicen esta circunstancia, constatando el desgaste producido por los años (Llorent y Ruiz-Calzado, 2016), puntualizando que la IE adquirida evoluciona con el paso del tiempo (Suriá, 2017).

Con respecto al tercer objetivo, predecir que variables de la IE son las que explican el optimismo vs. pesimismo de los sujetos a estudio, los resultados evidencian que la variable de IE (reparación) entró en el modelo de regresión para explicar la disposición al optimismo. El análisis de regresión múltiple realizado explicó un $24,1 \%$ de la variabilidad. Para explicar qué variables de IE predijeron la disposición al pesimismo, los resultados reflejaron que la variable de IE (manejo del estrés) entró en el modelo de regresión explicando el $24,4 \%$ de la varianza. Es decir, las personas que son capaces de gestionar y regular sus estados emocionales tienen un mayor bienestar, presentando una tendencia más optimista sobre eventos futuros (Cejudo et al., 2018; Moya-Smith, 2018). Por el contrario, las personas pesimistas tienen dificultades para afrontar el estrés de manera adecuada (Carver y Scheier, 2014). Este dato es relevante porque pone de manifiesto el valor de la IE para modular los niveles de optimismo vs. pesimismo del individuo en situaciones adversas, facilitando el impacto sobre una situación desfavorable con estrategias emocionales adecuadas.

En conclusión, los resultados obtenidos determinan la necesidad de incluir programas de IE en centros asistenciales para personas con discapacidad intelectual. Potenciar la adquisición de competencias emocionales en los profesionales repercutirá de manera significativa en un buen desarrollo de la labor, incrementando los niveles de eficacia, circunstancia fundamental si tenemos en cuenta la repercusión de las acciones en colectivos con características diferenciadas.

No obstante, hay que mencionar algunas limitaciones en este trabajo. En primer lugar, el hecho de trabajar con una muestra pequeña y en un contexto transversal, minimiza la posibilidad de generalizar resultados. Sería necesario replicar este modelo en contextos longitudinales, con el propósito de evaluar la IE en la predicción de optimismo vs. pesimismo. En segundo lugar, la proporción entre mujeres y hombres no es equilibrada en nuestra muestra ( $81,40 \%$ mujeres y $18,60 \%$ hombres), pudiendo resultar contradictorios los datos por género. Por último, se debería concretar la relación entre IE habilidad y rasgo en cada uno de los ítems con el fin de evidenciar la concordancia entre instrumentos.

A pesar de las limitaciones expuestas, este trabajo aporta datos relevantes sobre las relaciones entre IE habilidad y rasgo con optimismo vs. pesimismo en profesionales que atienden a personas con discapacidad intelectual, contribuyendo de manera fehaciente al diseño y desarrollo de nuevas estrategias para la prevención del agotamiento y desgaste profesional, siendo su proyección necesaria al colectivo de personas con discapacidad intelectual a cargo.

De igual forma, aunque se calculó la fiabilidad y consistencia interna en los instrumentos empleados, se sugiere llevar a cabo el empleo poco utilizado del coeficiente Omega para futuras investigaciones, con el propósito de obtener una medida más precisa. 


\section{Referencias bibliográficas}

Aradilla-Herrero, A. et al. (2014). "Perceived emotional intelligence in nursing: psychometric properties of the Trait Meta-Mood Scale". Journal of Clinical Nursing, 23, pp. 955-966. DOI: http://dx.doi.org/10.1111/jocn.12259.

Ato, M. et al. (2013). "Un sistema de clasificación de los diseños de investigación en psicología”. Anales de Psicología, 29(3), pp. 1038-1059. DOI: https://doi.org/10.6018/analesps.29.3.178511.

Augusto-Landa, J. et al. (2011). "Inteligencia emocional percibida y estrategias de afrontamiento al estrés en profesores de enseñanza primaria: propuesta de un modelo explicativo con ecuaciones estructurales (SEM)". Revista de Psicología Social, 26, pp. 413-425. DOI: https://doi.org/10.1174/021347411797361310.

Bar-On, R. (1997). The Emotional Quotient Inventory (EQ-i). A test of Emotional Intelligence. Toronto: Multi-Health Systems.

Bar-On, R., y Parker, J. D. (2000). The emotional quotient invetitorv: Youth version (EQ-i:YV). North Tonawanda, Nueva York: Multi-Health Systems.

Bar-On, R. (2006). "The Bar-On model of emotional-social intelligence (ESI)”. Psicothema, 18(suppl.), pp. 13-25.

Blas, V. (2016). La inteligencia optimista. Buenos Aires: V\&R Editoras.

Carver, C. S. y Scheier, M. F. (2014). “Dispositional optimism”. Trends in Cognitive Sciences, 18(6), pp. 293-299. DOI: https://doi.org/10.1016/j.tics.2014.02.003.

Cazalla-Luna, N. y Molero, D. (2016). "Inteligencia emocional percibida, disposición al optimismo-pesimismo, satisfacción vital y personalidad de docentes en su formación inicial”. Revista de Investigación Educativa, 34(1), pp. 241-258. DOI: https://doi.org/10.6018/rie.34.1.220701.

Cazalla-Luna, N. y Molero, D. (2018). "Emociones, afectos, optimismo y satisfacción vital en la formación inicial del profesorado". Profesorado, Revista de Currículum y Formación del Profesorado, 22(1), pp. 215-233.

Cejudo, J.et al. (2016). "Inteligencia emocional y resiliencia: su influencia en la satisfacción con la vida de estudiantes universitarios”. Anuario de Psicología, 46, pp. 51-57. DOI: https://doi.org/10.1016/j.anpsic.2016.07.001.

Cejudo, J. et al. (2018). "Emotional intelligence and its relationship with levels of social anxiety and stress in adolescents”. Int. J. Environ. Res. Public Health, 15(6), pp. 1073.

Cohen, J. (1988). Statistical power analysis for the behavioral sciencies. New York: Erlbaum.

Domínguez-Lara, S. A. D. y Merino-Soto, C. M. (2015). “¿Por qué es importante reportar los intervalos de confianza del coeficiente alfa de Cronbach?”. Revista Latinoamericana de Ciencias Sociales, Niñez y Juventud, 13(2), pp. 1326-1328.

Extremera, N. et al. (2005). "La inteligencia emocional percibida y su influencia sobre la satisfacción vital, la felicidad subjetiva y el engagement en trabajadores de centros para personas con discapacidad intelectual". Ansiedad y Estrés, 11(1), pp. 63-73.

Feldman, D. B. y Kubota, M. (2015). "Hope, self-efficacy, optimism, and academic achievement: Distinguishing constructs and levels of specificity in predicting college grade-point average". Learning and Individual Differences, 37, pp. 210-216. DOI: http://dx.doi.org/10.1016/j.lindif.2014.11.022. 
Fernández-Berrocal, P. et al. (2004). "Validity and reliability of the Spanish modified version of the Trait Meta-Mood Scale". Psychological Reports, 94, pp. 751-755. DOI: https://doi.org/10.2466/pr0.94.3.751-755.

Fernández-Berrocal, P. y Extremera, N. (2006). "Emotional intelligence: A theoretical and empirical overview of its first 15 years of history". Psicothema, 18, pp. 7-12.

Fernández-Berrocal, P. et al. (2018). "The relationship of Botín Foundation's Emotional Intelligence Test (TIEFBA) with personal and scholar adjustment of Spanish”. Rev. Psicodidáct., 23, pp. 1-8. DOI: https://doi.org/10.1016/j. psicod.2017.07.001.

Gil-Pascual, J. A. (2015). "Análisis estadísticos básicos. Cuándo y cuáles emplear”. En A. Pantoja (coord.), Manual básico para la realización de tesinas, tesis y trabajos de investigación (pp. 342-359). Madrid: EOS Universitaria.

Gillham, J. et al. (2002). "Building optimism and preventing depressive symptoms in children". En E. C. Chang (ed.), Optimism and Pessimism. Implications for theory, research, and practice (pp. 321-346). Washington, DC: APA.

Giménez, M. (2005). “Optimismo y pesimismo. Variables asociadas en el contexto escolar”. Pulso, 28, pp. 9-25.

Gómez-Ortiz, O. et al. (2016). "Parenting styles and bullying. The mediating role of parental psychological aggression and physical punishment”. Child Abuse \& Neglect, 51, pp. 132-143. DOI: https://doi.org/10.1016/j. chiabu.2015.10.025.

Huerta-Ramírez, Y. y Rivera-Heredia, M. E. (2017). "Resiliencia, recursos familiares y espirituales en cuidadores de niños con discapacidad". Journal of Behavior, Health \& Social Issues, 9(2), pp. 70-81. DOI: https://doi. org/10.1016/j.jbhsi.2018.01.005.

Jöreskog, KG. (1971). "Statistical analysis of sets of congeneric tests". Psychometrika, 36, pp. 109-133. DOI: https://doi.org/10.1007/BF02291393.

Knowlden, A. P. et al. (2016). "Lifestyle and mental health correlates of psychological distress in college students". Health Education Journal, 75, pp. 370-382. DOI: https://doi.org/10.1177/0017896915589421.

Lara, F. J. P. et al. (2014). "Psychological modulation in patients surgically intervened for gastroesophageal reflux disease". Diseases of the Esophagus, 27, pp. 538-546.

Latorre, A. et al. (2003). Bases metodológicas de la investigación educativa. Barcelona: Ediciones Experiencia.

López-Fernández, C. (2015). "Inteligencia emocional y relaciones interpersonales en los estudiantes de enfermería”. Educación Médica, 16, pp. 83-92. DOI: https://doi.org/10.1016/j.edumed.2015.04.002.

López-Zafra, E. et al. (2014). "EQi Versión Corta (EQI-C). Adaptación y validación al español del EQ-i en universitarios". Boletín de Psicología, 110, pp. 21- 36.

Llorent, V.J. y Ruiz-Calzado, I. (2016). "Burnout and its relation to sociodemographic variables among education professionals working with people with disabilities in Córdoba (Spain)". Revista Ciência \& Saúde Coletiva, 21(10), pp. 3287-3295. DOI: https://doi.org/10.5944/educxx1.15459.

Mayer, J. D. et al. (2001). "Emotional intelligence as a standard intelligence”. Emotion, 1(3), pp. 232-242. DOI: https://doi.org/10.1037/1528-3542.1.3.232.

Mayer, J. D. et al. (2008). "Emotional intelligence: New ability or eclectic traits?". The American Psychologist, 63, pp. 503-517. DOI: https://doi.org/10.1037/0003-066X.63.6.503.

Mayer, J. D. et al. (2016). "The ability model of emotional intelligence: Principles and updates". Emotion Review, 8(4), pp. 290-300. DOI: https://doi.org/10.1177/1754073916639667. 
McDonald, R. P. (1999). Test theory: A unified treatment. Mahwah: Lawrence Erlbaum Associates, Inc.

Montero, X. et al. (2014). "Carga, ansiedad y depresión en cuidadores primarios informales de niños con cáncer". Psicología y Salud, 24(1), pp. 45-53.

Morales-Rodríguez, F. M. (2017). "Relaciones entre afrontamiento del estrés cotidiano, autoconcepto, habilidades sociales e inteligencia emocional”. European Journal of Education and Psychology, 10(2), pp. 41-48. DOI: https://doi.org/10.1016/j.ejeps.2017.04.001.

Moya-Smith, L. (2018). "Optimismo disposicional y estilos de vida promotores de salud en estudiantes de Ciencias de la Salud”. Revista española de enfermería de salud mental, 3, pp. 3-23.

Munkholm, K. et al. (2012). "State-related alterations of gene expression in bipolar disorder: a systematic review". Bipolar Disorders, 14, pp. 684-696. DOI: https://doi.org/10.1111/bdi.12005.

Nespereira-Campuzano, T. y Vázquez-Campo, M. (2017). "Emotional intelligence and stress management in Nursing professionals in a hospital emergency department". Enfermería Clínica, 27(3), pp. 172-178. DOI: https:// doi.org/10.1016/j.enfcli.2017.02.007.

O’Boyle, E. H. et al. (2011). "The relation between emotional intelligence and job performance: A meta-analysis". Journal of Organizational Behavior, 32(5), pp. 788-818. DOI: https://doi.org/10.1002/job.714.

Ortiz, M. S. et al. (2016). "Validación de la versión en español de la Escala de Optimismo Disposicional (LOT-R) en una muestra chilena de estudiantes universitarios". Terapia Psicológica, 34(1), pp. 53-58. DOI: https://doi. org/10.4067/S0718-48082016000100006.

Peterson, C. y Seligman, M. E. (1984). "Causal explanations as a risk factor for depression: Theory and evidence". Psychological Review, 91(3), pp. 347-374. DOI: https://doi.org/10.1037/0033-295X.91.3.347.

Pino, T. et al. (2016). "La eficacia de la reexperimentación emocional: el papel del optimismo y la alexitimia". European Journal of Investigation in Health, Psychology and Education, 6, pp. 193-205. DOI: https://doi. org/10.30552/ejihpe.v6i3.179.

Pulido-Acosta, F. y Herrera-Clavero, F. (2018). "Relaciones entre rendimiento e inteligencia emocional en secundaria”. Tendencias Pedagógicas, 31, pp. 165-185.

Remor, E. et al. (2006). "El optimismo y la experiencia de ira en relación con el malestar físico". Anales de Psicología, 22(1), pp. 37-44.

Salovey, P. et al. (1995). "Emotional attention, clarity and repair: exploring emotional intelligence using the Trait Meta-Mood Scale". En J. W. Pennebaker (ed.), Emotion, disclosure and health (pp. 125-154). Washington: American Psychological Association.

Salovey, P. y Mayer, J. D. (1990). "Emotional intelligence”. Imagination, Cognition y Personality, 9(3), pp. 185-211. DOI: https://doi.org/10.2190/DUGG-P24E-52WK-6CDG.

Sanin, A. y Salanova-Soria, M. (2018). "Verificación de las condiciones psicométricas del Test de Orientación en la Vida (LOT-R) aplicado en población trabajadora colombiana”. Revista Interamericana de Psicología Ocupacional, 35(2), pp. 58-67. DOI: https://doi.org/10.21772/ripo.v35n2a01.

Stone, L. L. et al. (2015). "The Strengths and Difficulties Questionnaire: psychometric properties of the parent and teacher version in children aged 4-7”. BMC Psychology, 3(1), pp. 1-12. DOI: https://doi.org/10.1186/s40359015-0061-8. 
Suriá, R. (2017). "Redes virtuales y apoyo social percibido en usuarios con discapacidad: análisis según la tipología, grado y etapa en la que se adquiere la discapacidad". Escritos de Psicología, 10, pp. 31-40. DOI: https:// doi.org/10.5231/psy.writ.2017.21403.

Tolegenova, A. A. et al. (2014). "Trait meta-mood, gender and EEG response during emotion-regulation”. Personality and Individual Differences, 65, pp. 75-80. DOI: https://doi.org/10.1016/j.paid.2014.01.028.

Veloso-Besio, C. et al. (2013). "Relación entre inteligencia emocional, satisfacción vital, felicidad subjetiva y resiliencia en funcionarios de Educación Especial”. Estudios Pedagógicos, 39(2), pp. 355-366. DOI: http://dx.doi. org/10.4067/s0718-07052013000200022.

Villardefrancos, E. et al. (2012). "Estrés en profesores de enseñanza secundaria: un análisis desde el optimismo". European Journal of Investigation in Health, Psychology and Education, 2, pp. 91-101. DOI: https://doi. org/10.30552/ejihpe.v2i3.29.

Vizoso-Gómez, C. y Arias-Gundín, O. (2018). "Resiliencia, optimismo y burnout académico en estudiantes universitarios". European Journal of Education and Psychology, 11(1), pp. 47-59. DOI: https://doi.org/10.30552/ejep. v11i1.185.

Xia, J. et al. (2016). "Chinese version of the Optimism and Pessimism Scale: Psychometric properties in mainland China and development of a short form". Journal of Health Psychology, 21(6), pp. 983-993. DOI: https://doi. org/10.1177/1359105314544071.

Zych, I. et al. (2016). "Competencias socio-emocionales para la convivencia y la ciberconvivencia”. En F. CórdobaAlcaide et al. (eds.), Gestión de la convivencia y afrontamiento de la conflictividad escolar y el bullying (pp.5168) Santiago, Chile: Ril. 